\title{
Phase Separations in the Narrow-Bandwidth Limit of the Penson-Kolb-Hubbard Model at Zero Temperature
}

\author{
K.J. KAPCIA ${ }^{a, b, *}$ AND W.R. CZART ${ }^{c}$ \\ ${ }^{a}$ Institute of Physics, Polish Academy of Sciences, Aleja Lotników 32/46, 02-668 Warsaw, Poland \\ ${ }^{b}$ Institute of Nuclear Physics, Polish Academy of Sciences, W.E. Radzikowskiego 152, 31-342 Kraków, Poland \\ ${ }^{c}$ Faculty of Physics, Adam Mickiewicz University, ul. Umultowska 85, 61-614 Poznań, Poland \\ In this work we study the ground state of the Penson-Kolb-Hubard model in the limit of narrow-bandwidth. \\ We present phase diagrams of the model for fixed chemical potential and concentration (involving various phase \\ separations). The results are derived within the Hartree-Fock approximation (HFA) in the narrow-bandwidth \\ regime and compared with the exact ones in the atomic limit and the high-dimension regime. Our investigation \\ reveals that the HFA can reconstruct the exact diagram at the ground state when the bandwidth approaches to \\ zero.
}

DOI: 10.12693/APhysPolA.133.401

PACS/topics: 71.10.Fd, 71.10.Hf, 74.20.-z, 74.25.Dw, 64.75.Gh

\section{Introduction}

The unconventional superconductivity (SC) is still very interesting and intensively investigated phenomenon due to its relevance to many groups of materials [1-4]. One of the simplest paradigmatic models for superconductivity with extremely short coherence length is the PensonKolb-Hubbard (PKH) model [5-13]. Its hamiltonian can be written in the following form:

$$
\begin{aligned}
& \hat{H}=\frac{t}{\sqrt{z}} \sum_{\langle i, j\rangle, \sigma}\left(\hat{c}_{i \sigma}^{+} \hat{c}_{j \sigma}+h . c\right)+U \sum_{i} \hat{n}_{i \uparrow} \hat{n}_{i \downarrow} \\
& -\frac{2 I}{z} \sum_{\langle i, j\rangle} \hat{\rho}_{i}^{+} \hat{\rho}_{j}^{-}-\mu \sum_{i} \hat{n}_{i},
\end{aligned}
$$

where $\hat{n}_{i}=\sum_{\sigma} \hat{n}_{i \sigma}, \quad \hat{n}_{i \sigma}=\hat{c}_{i \sigma}^{+} \hat{c}_{i \sigma}, \quad \hat{\rho}_{i}^{+}=\left(\hat{\rho}_{i}^{-}\right)^{\dagger}=\hat{c}_{i \uparrow}^{+} \hat{c}_{i \downarrow}^{+}$; $\hat{c}_{i \sigma}\left(\hat{c}_{i \sigma}^{+}\right)$denotes the annihilation (creation) operator of an electron with spin $\sigma=\uparrow, \downarrow$ at the site $i . \sum_{\langle i, j\rangle}$ indicates the sum over nearest-neighbour sites $i$ and $j$ independently. $z$ denotes the number of the nearest neighbours. The first term of the above Hamiltonian is the kinetic term (with electron hopping only between nearestneighbour sites), followed by the on-site interaction term $U$ and intersite pair-hopping interaction $I$. Finally, $\mu$ is the chemical potential, dependent on the concentration of electrons: $n=\frac{1}{N} \sum_{i}\left\langle\hat{n}_{i}\right\rangle$, and $N$ is the total number of lattice sites $(0 \leqslant n \leqslant 2)$.

It is worth mentioning that in spite of very intensive studies of the Hubbard model (the PKH model with $I=0)$ exact solutions for this model exist only for one dimension $(d=1)$ [14] and $d \rightarrow+\infty$ [15]. Further more, for the Penson-Kolb model, an electronic model with intersite interactions only $(U=0)$, within the broken-symmetry Hartree-Fock (mean-field) approx-

*corresponding author; e-mail: konrad.kapcia@ifpan.edu.pl imation (HFA), one obtains exact results in the limit $d \rightarrow+\infty$ at any $T \geqslant 0[16]$. Otherwise, for $d<\infty$ at $T>0$, the HFA is significantly less reliable. It is particularly unreliable in the limit of strong coupling and lowdimensions as it neglects phase fluctuations and shortrange correlations. So far the PKH model has been investigated only in a few particular limits [6-9]. The main efforts concerned the ground state (i.e. at $T=0$ ) properties of the model in $d=1$ at half-filling [6-8]. For $d=+\infty$ hypercubic lattices the ground state diagrams of the PKH model for both signs of $I$ and $U$ were also determined by means of the HFA [8-13].
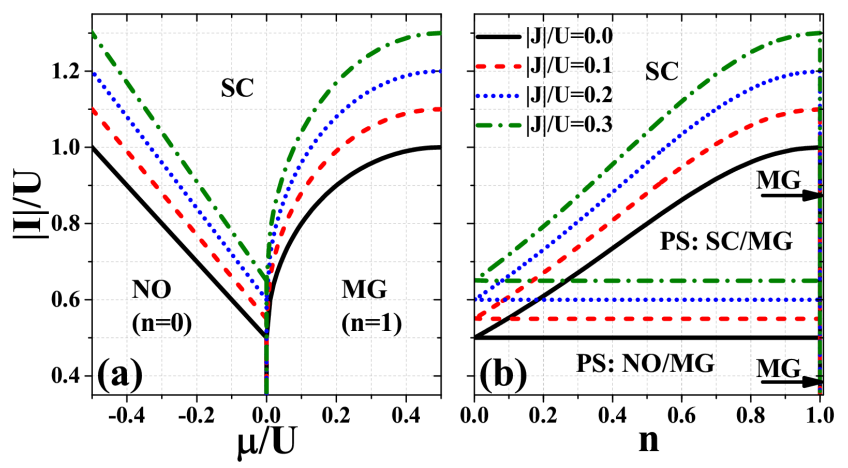

Fig. 1. Ground state diagrams of Hamiltonian $\hat{H}_{A L}$ (atomic limit, $t=0$ ) for a few $|J|$ (as labelled) as a function of $\mu$ (a); and as a function of $n(\mathrm{~b})$. It is an exact result for $d \rightarrow+\infty$.

It was proven that all interactions between particles on different sites get trivial in limit $d \rightarrow+\infty[17,18]$ and may be treated in the (Hartree) mean-field approximation. The Hubbard on-site $U$ interaction is the only interaction which remains dynamical at this limit $[15,17]$. Thus, a question about an accuracy of the standard HFA approximation for the $U$ term is still relevant. In our previous work [12] we presented the $T=0$ phase dia- 
grams, as a function of $n$, involving only homogeneous phases and we have compared those results with the exact solutions (for $d \rightarrow+\infty$ ) obtained for the atomic limit $(t=0)$. In this work we extend our previous investigation of the diagrams by taking into consideration also the phase separated (PS) states. Due to the electronhole symmetry of the studied PKH model the diagrams presented are symmetric with respect to the half-filling ( $n=1$ or $\mu / U=1 / 2$, equivalently).
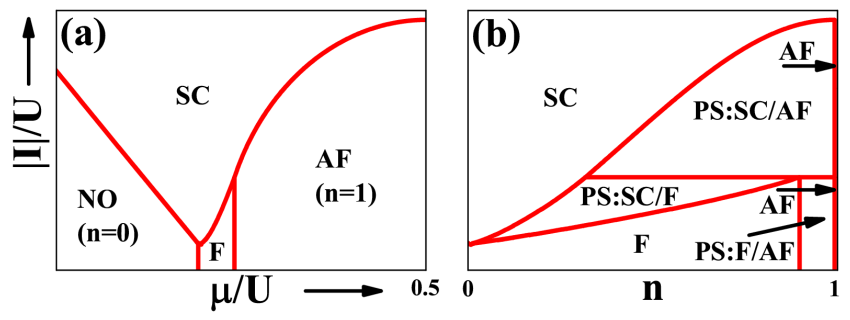

Fig. 2. Schematic $T=0$ diagrams of the PKH model for small $t \neq 0$ as a function of $\mu(\mathrm{a})$; and as a function of $n(\mathrm{~b})$.

\section{Results}

First, we discuss the atomic limit of the PKH model. For $t=0$ one can derive rigorous results for $d \rightarrow \infty[9,19]$. Due to the fact that for $U,|I| \gg|t|$ effective magnetic interaction $J_{i j}^{e f f} \approx-4 t_{i j}^{2} /(U-|I|)$ appears in the system, we additionally introduce the isotropic magnetic term into our considerations $\hat{H}_{M}=-(2 J / z) \sum_{\langle i, j\rangle} \hat{\boldsymbol{s}}_{i} \cdot \hat{\boldsymbol{s}}_{j}$, where $\hat{\boldsymbol{s}}_{i}$ is an operator of total electron spin at $i$ site. The ground state phase diagrams for Hamiltonian $\hat{H}_{A L}=$ $\hat{H}(t=0)+\hat{H}_{M}$ for several values of $J$ are shown in Fig. 1 (cf. Refs. [19,12] and references therein). The model $\hat{H}_{A L}$ exhibits symmetry $I \leftrightarrow-I$ and for $I>0$ the $s$-wave SC (SS) occurs for sufficiently large $|I|$, whereas for $I<0$ the $\eta$-wave $\mathrm{SC}(\eta \mathrm{S})$ is stable. For lower $|I|$ the non-ordered (NO) phase or the magnetic (MG) phase is present, which can be either ferromagnetic (F) for $J>0$ or antiferromagnetic $(\mathrm{AF})$ for $J<0$. The $\mathrm{SC}-\mathrm{MG}$ boundary is discontinuous for fixed $\mu$ (Fig. 1a) and the PS state occurs: $\mathrm{PS}: \mathrm{SC} / \mathrm{MG}$ in definite range of $n$ (it is a macroscopic coexistence of the SC and MG phases, Fig. 1b). The MG-NO boundary is also discontinuous, whereas the NO-SC transition is continuous. For $|J|=0$ the MG phase changes into the non-ordered (NO') phase and the PS:NO/NO' state is degenerate with homogeneous NO' phase $[12,19]$.

For $t \neq 0$ the PKH model does not exhibit symmetry $I \leftrightarrow-I$. Even so, for small $t \neq 0$ the structure of the phase diagrams for both signs of $I$ interaction are qualitatively similar. For $U>0$ they are shown schematically in Fig. 2. The main qualitative difference with respect to the atomic limit is that both MG phases can occur, which are favoured by strong enough $U /|I|$. The presence of the AF phase at half-filling is not surprising due to the fact that $J_{i j}^{e f f}<0(U>|I|)$, but also the F phase
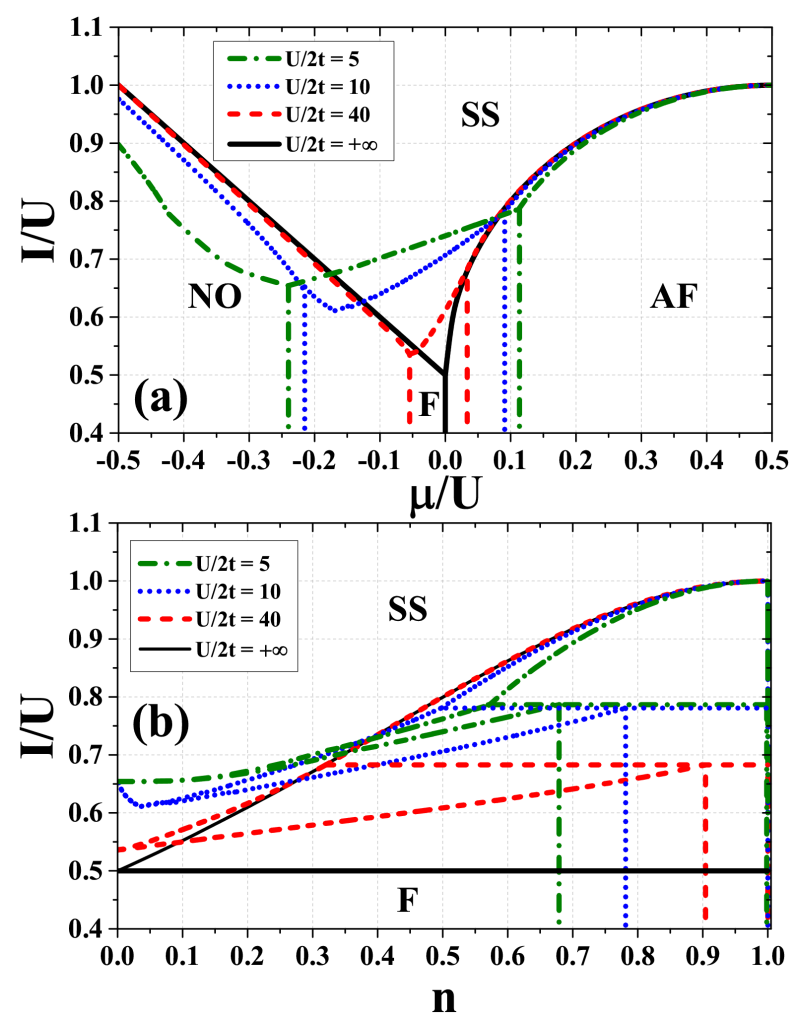

Fig. 3. Phase diagrams for $t \neq 0$ and $I>0$ : (a) $I / U$ vs. $\mu / U$ and (b) $I / U$ vs. $n$ (involving phase separations). A few fixed values of $U$ interaction are shown (as labelled). The structures of the diagrams are shown in Fig. 2.

is stable away from half-filling. The NO-F and NO-SC transitions are continuous (fixed $\mu / U$ or $n=0$ ). The $\mathrm{SC}$ phases can occur for any $n$, if $|I| / U$ is sufficiently large. The $\mathrm{F}-\mathrm{AF}$ (not dependent on $\mu$ ), $\mathrm{SC}-\mathrm{F}$, and $\mathrm{SC}-$ AF transitions are discontinuous for fixed $\mu$ (Fig. 2a) and thus different PS states can occur at fixed $n$ : PS:F/AF, PS:SC/F, PS:SC/AF (Fig. 2b). The transitions between the PS states with increasing $|I| / U$ for fixed $n$ (horizontal line in Fig. 2b), are associated with a discontinuity of the order parameters in one of the domains in the PS state.

In the following we discuss quantitative changes of phase diagrams for $U>0$ and small $t \neq 0$. The semielliptical density of states (SE-DOS) is used: $D(\epsilon)=$ $\frac{1}{2 \pi t^{2}} \sqrt{4 t^{2}-\epsilon^{2}}$ for $\epsilon<2 t$, where $2 t$ is half-bandwidth, however, the shape of DOS in the narrow-band limit investigated here does not affect the results qualitatively. Similarly as in Ref. [12], we use the HFA for $U$ term (with electron Wick theorem for averages of fermion operators) including Hartree (density-density), Fock (magnetic) and anomalous (superconducting) terms. For $I$ term, as for the atomic limit, only Hartree decoupling is applied.

Figures 3 and 4 present the ground state phase diagrams for $I>0$ and $I<0$, respectively, which are plotted for several fixed positive values of $U$ interaction: $U / 2 t=5,10,40$ (dashed-dotted, dotted, and dashed lines, respectively) and for $U / 2 t \rightarrow+\infty$ (exact result, 

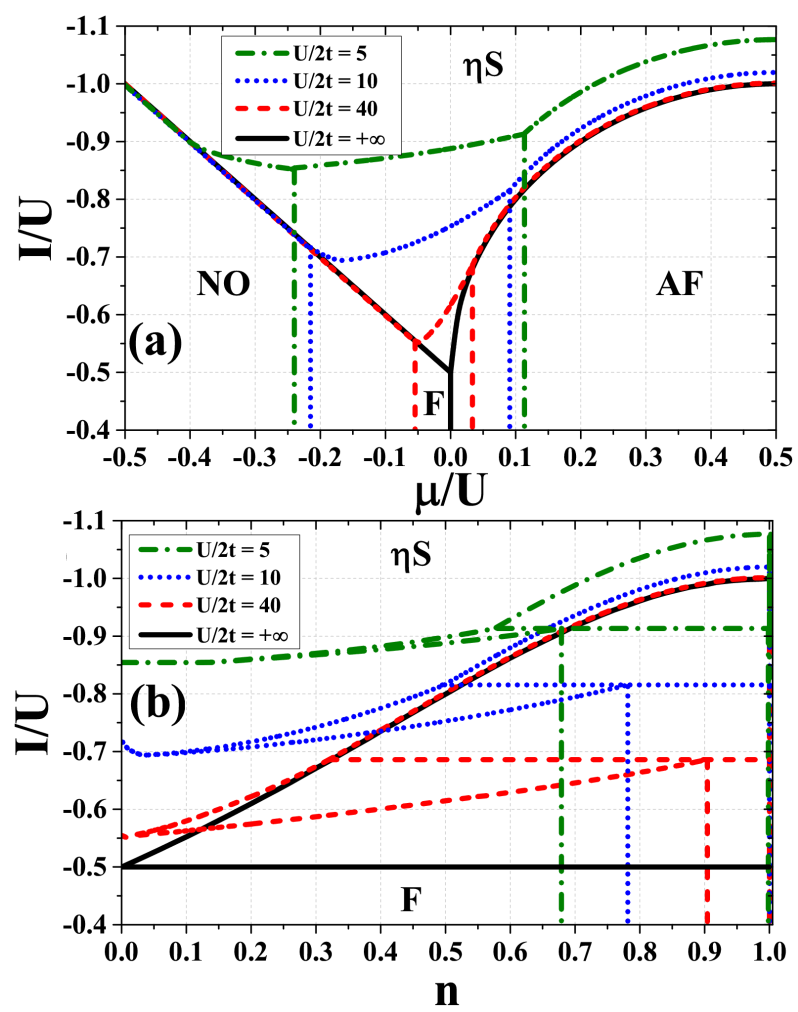

Fig. 4. Phase diagrams for $t \neq 0$ and $I<0$ : (a) $I / U$ vs. $\mu / U$ and (b) $I / U$ vs. $n$ (involving phase separations). A few fixed values of $U$ interaction are shown (as labelled). The structures of the diagrams are shown in Fig. 2.

solid lines). With increasing $U / 2 t$ the transition lines for $t \neq 0$ approach rigorous results obtained at the atomic limit. The phase boundaries at the atomic limit can be considered as a continuous limit of the HFA results for $t \rightarrow 0$ with infinitesimal but still finite $t$, which introduces magnetic orderings as well as $U / 2 t$-dependent competition between $\mathrm{F}$ and $\mathrm{AF}$ orderings. In Figs. 3b and $4 \mathrm{~b}$, one observes that with increasing $U / 2 t$ the area occupied by the PS states involving the $\mathrm{F}$ phase shrinks and disappears at $t \rightarrow 0$. In this process, with suppressing the $\mathrm{F}$ phase (towards smaller values of $|I| / U$ ), the $n$-independent F-PS:F/AF boundary shifts towards $n=1$. At the same time, with increasing $U / 2 t$, in Figs. 3a and 4a, $\mu$-ranges occupied by the NO and AF phases widen, whereas the area of the $\mathrm{F}$ phase occurrence vanishes. It is associated with an extension of a range of $n$, in which the homogeneous $\mathrm{F}$ phase is stable (for low $|I| / U)$. Notice that with increasing $U / 2 t$ the region of the PS:SC/AF state expands. Moreover, the SS-AF boundary is almost independent on $U / 2 t$ (near $\mu \approx U / 2$ ), whereas for $t \neq 0$ the $\eta \mathrm{S}-\mathrm{AF}$ boundary is shifted towards higher $|I| / U$. On the other hand, a location of the continuous $\eta \mathrm{S}-\mathrm{NO}$ line is not dependent on $U / 2 t$ for small $\mu \ll 0$, but the NO-SS boundary for $t \neq 0$ is located at lower $|I| / U$ (cf. also Ref. [12]).

\section{Conclusion}

To conclude, in this work we investigated the PKH model for small, but finite single-electron hopping. We showed that the HFA decoupling of the $U$ term in the limit $t \rightarrow 0$ gives reasonable results, which are in coincidence with the exact results for $t=0$ (in the limit $d \rightarrow+\infty$ ) at least at $T=0$. The results obtained within both approaches are consistent, although in the case of the finite single-electron hopping, the phases with magnetic long-range order also are stable. Obviously, the HFA approach used in this work would largely overestimate critical temperatures. Here, we only discussed the phase diagrams determined by a difference between grand canonical potentials (energies) of the states near the boundaries and we did not analyse the behaviour of the thermodynamical potentials with varying model parameters. Notice that the macroscopically PS states considered in this work are specific for short-range interactions. The longer-range interactions prevent such phase separations and other states with a formation of various textures are possible (cf. also e.g. Ref. [13]).

\section{Acknowledgments}

The authors are indebted to Stanisław Robaszkiewicz and Andrzej Ptok for very fruitful discussions during the course of this work. K.J.K. acknowledges the support from the National Science Centre (NCN, Poland) under grants no. UMO-2016/21/D/ST3/03385 and no. $2017 / 24 / \mathrm{C} / \mathrm{ST} 3 / 00276$.

\section{References}

[1] R. Micnas, J. Ranninger, S. Robaszkiewicz, Rev. Mod. Phys. 62, 113 (1990); M. Imada, A. Fujimori, Y. Tokura, Rev. Mod. Phys. 70, 1039 (1998).

[2] D.C. Johnston, Adv. Phys. 59, 803 (2010); P.M. Aswathy, J.B. Anooja, P.M. Sarun, U. Syamaprasad, Supercond. Sci. Technol. 23, 073001 (2010); G.R. Stewart, Rev. Mod. Phys. 83, 1589 (2011).

[3] M.M. Wysokiński, M. Abram, J. Spałek, Phys. Rev. B 90, 081114(R) (2014); M. Abram, M.M. Wysokiński, J. Spałek, J. Magn. Magn. Mater. 400, 27 (2016).

[4] J. Spałek, M. Zegrodnik, J. Kaczmarczyk, Phys. Rev. B 95, 024506 (2017); M. Abram, M. Zegrodnik, J. Spałek, J. Phys.: Condens. Matter 29, 365602 (2017).

[5] A. Belkasri, F.D. Buzatu, Phys. Rev. B 53, 7171 (1996); F. Dolcini, A. Montorsi, Phys. Rev. B 62, 2315 (2000).

[6] A. Hui, S. Doniach, Phys. Rev. B 48, 2063 (1993); B. Bhattacharyya, G.K. Roy, J. Phys. Condens. Matter 7, 5537 (1995).

[7] G.I. Japaridze, E. Müller-Hartmann, J. Phys.: Condens. Matter 9, 10509 (1997); G.I. Japaridze, A.P. Kampf, M. Sekania, P. Kakashvili, Ph. Brune, Phys. Rev. B 65, 014518 (2001). 
[8] S. Robaszkiewicz, B.R. Bułka, Phys. Rev. B 59, 6430 (1999).

[9] S. Robaszkiewicz, W. R. Czart, Acta Phys. Pol. B 32, 3267 (2001); W.R. Czart, S. Robaszkiewicz, Acta. Phys. Pol. A 106, 709 (2004).

[10] A. Ptok, M. Maśka, M. Mierzejewski, J. Phys.: Condens. Matter 21, 295601 (2009); A. Ptok, K.J. Kapcia, Supercond. Sci. Technol. 28, 045022 (2015); A. Ptok, J. Phys. Condens. Matter 29, 475901 (2017).

[11] W.R. Czart, S. Robaszkiewicz, Acta Phys. Pol. A 127, 275 (2015); W.R. Czart, S. Robaszkiewicz, Acta Phys. Pol. A 127, 278 (2015).

[12] K.J. Kapcia, W.R. Czart, Acta Phys. Pol. A 130, 617 (2016).
[13] K.J. Kapcia, W.R. Czart, A. Ptok, J. Phys. Soc. Jpn. 85, 044708 (2016); W.R. Czart, S. Robaszkiewicz, Acta Phys. Pol. A 130, 613 (2016).

[14] E.H. Lieb, F.Y. Wu, Phys. Rev. Lett. 20, 1445 (1968).

[15] A. Georges, G. Kotliar, W. Krauth, M.J. Rozenberg, Rev. Mod. Phys. 68, 13 (1996)

[16] W.R. Czart, S. Robaszkiewicz, Phys. Rev. B 64 104511 (2001); M. Mierzejewski, M.M. Maśka, Phys. Rev. B 69, 054502 (2004).

[17] E. Müller-Hartmann, Z. Phys. B 74, 507 (1989).

[18] P.A. Pearce, C.J. Thompson, Commun. Math. Phys. 41, 191 (1975); P.A. Pearce, C.J. Thompson, Commun. Math. Phys. 58, 131 (1978).

[19] K.J. Kapcia, J. Supercond. Nov. Magn. 28, 1289 (2015). 